\title{
Fabricating Flaps in the Forearm Prior to Tracheal Reconstruction
}

\author{
Gautam Biswas ${ }^{1}$ Karnav Bharat Panchal ${ }^{1}$ Prateek V. Jain ${ }^{2}$ Kapila Manikantan ${ }^{2}$ \\ Rajeev Sharan² Pattatheyil Arun²
}

${ }^{1}$ Department of Plastic and Reconstructive Surgery, TATA Medical Center, Kolkata, West Bengal, India

${ }^{2}$ Department of Head and Neck Surgery, TATA Medical Center, Kolkata, West Bengal, India

\begin{abstract}
Address for correspondence Gautam Biswas, MS, Mch, DNB, Department of Plastic and Reconstructive Surgery, TATA Medical Center, 14 major ARTERIAL Road (EW), New Town Rajarhat, Kolkata 700156, West Bengal, India (e-mail: dr.biswas@gmail.com).
\end{abstract}

Indian J Plast Surg:2021;54:53-57.

\begin{abstract}
Background The process of reconstruction of tracheal defects is complex and still not optimum. Options range from using staged reconstructions, combining flaps with autologous or alloplastic implants, as well as use of tissue-engineered constructs combined with vascularized tissues which are lined with cell cultures. Staged reconstructions using prelaminated epithelium, and prefabricated flaps, help in reconstruction of this complex structure. Prefabricating the flap at a different site allows for integration of the tissues prior to its transfer.

Method This article reports two patients planned for tracheal reconstruction for the purpose of advanced papillary carcinoma of the thyroid invading the trachea. Staged reconstruction using a prefabricated radial artery forearm flap (RAFF) and split rib cartilage was performed. In the second patient, a young girl, a similar construct of the RAFF, prelaminated with buccal mucosa, was performed. However, in the latter case, an intraoperative decision by the head and neck team to limit excision of the

Keywords

- tracheal reconstruction

- prefabricated flap

- prelaminated flap trachea sparing the mucosa was taken; the reconstruct in the forearm was redundant and needed to be discarded, replacing the defect with a free superficial circumflex iliac artery perforator (SCIP) flap.

Result At 3 years follow-up, both the patients are free of disease, with the construct serving its purpose in the older female.
\end{abstract}

\section{Introduction}

Tracheal reconstruction aims at constructing a semirigid structure, enough to withstand change in airway pressure without collapsing, with a well-mucosalized lining to prevent wound contraction.

Reconstruction of this complex structure has been described using skin flaps and placing implants, using either autologous or alloplastic material, to provide rigidity. Staged techniques lining a semirigid construct, mimicking tracheal

published online

November 30, 2020
DOI https://doi.org/

10.1055/s-0040-1721522 ISSN 0970-0358. epithelium, and using implanted cell-cultured ciliated epithelium have also been reported. ${ }^{1}$

\section{Material and Method}

\section{Patient 1}

A 52-year-old lady initially presented with papillary carcinoma, as reported by histopathology, following a partial thyroidectomy. She underwent completion thyroidectomy because of involved margins, followed by radioiodine therapy.

(C) 2020. Association of Plastic Surgeons of India.

This is an open access article published by Thieme under the terms of the Creative Commons Attribution-NonDerivative-NonCommercial-License, permitting copying and reproduction so long as the original work is given appropriate credit. Contents may not be used for commercial purposes, or adapted, remixed, transformed or built upon. (https://creativecommons.org/licenses/by-nc-nd/4.0/)

Thieme Medical and Scientific Publishers Pvt. Ltd. A-12, 2nd Floor, Sector 2, Noida-201301 UP, India 
The follow-up MRI at 3 months (-Fig. 1a) identified a nodular infiltrative hypoechoic mass $(2.4 \times 2.1 \times 1.2 \mathrm{~cm}$, approx. $3.1 \mathrm{~mL}$ ), which had infiltrated through the tracheal cartilage to present itself into the airway. Fiberoptic laryngoscopy showed a bulge in the tracheal mucosa.

A multidisciplinary team discussion plan involved a wide excision of the involved tracheal segment with staged reconstruction of the trachea.

\section{Planning}

The anticipated tracheal defect, according to the scans, extended from the cricoid cartilage to the third tracheal ring, involving the anterior two third of the trachea, sparing the posterior third, and measuring $4 \times 3 \mathrm{~cm}$. A staged prefabricated radial artery forearm flap (RAFF) with rib cartilages as a construct was planned.

\section{Surgical Execution}

In the first stage, prefabrication of the RAFF territory was done with split rib graft. A $6 \times 4 \mathrm{~cm}$ skin island was marked on the distal forearm skin overlying the radial vessels. The 7th rib including a portion of the cartilage was harvested from the left side of the chest and the graft was split into two strips.The split rib grafts were then inserted longitudinally in subcutaneous pockets on either side and parallel to the radial vascular pedicle through small incisions bluntly tunneled between the skin and fascia ( - Fig. 1b) After 4 weeks, the second stage was planned.

A low tracheostomy was done. Excision of the trachea was done, with the defect measuring $4 \times 3 \mathrm{~cm}$ and extending from the cricoid cartilage to the third tracheal ring along with anterior two thirds circumference of the trachea.

The fabricated forearm flap was transferred, matching the convexity of the tracheal ring, removing any excess portion of the construct, and stabilizing it to the present tracheal rings using 3-0 polydioxone suture ( - Fig. 1 c-d).

Flap monitoring was done using a laryngoscope daily for 2 days. Her tracheostomy was removed on the 24th postoperative day. Bronchoscopy done at the end of 1 and 3 months showed a well-healed flap (-Fig. 1e) The entire airway appeared clean, with no mucus accumulation. A CT scan neck done 36 months postsurgery showed no resorption with a maintained tracheal lumen (-Fig. 1f).

She lives a normal life, continues to tolerate a regular diet, and maintains a preoperative voice quality at the end of 45 months postreconstruction. There is no collapse or stenosis of the construct.

\section{Patient 2}

A 13-year-old girl earlier treated for papillary carcinoma of the thyroid 6 years prior. During follow-up imaging, a thyroid bed lesion involving the posterior wall of the trachea and also circumferentially involving the esophagus was observed ( - Fig. 2a). There were multiple bilateral lung nodules. Bronchoscopy did not show any mucosal involvement. Resection as planned by the head and neck team would entail segmental resection of trachea as well as a segment of cervical esophagus.

A staged reconstruction was planned, prefabricating a RAFF with rib cartilage, and this time, prelamination with buccal mucosa graft was planned and combined with a jejunum flap for the esophagus.

During the first stage, a RAFF was fabricated, partially elevated, and split ribs around $6 \mathrm{~cm}$ in length (-Fig. $\mathbf{2 b}$ ) were placed transversely between the fascia and skin

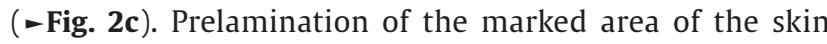
was done with two harvested strips of the buccal mucosa (-Fig. 2d).
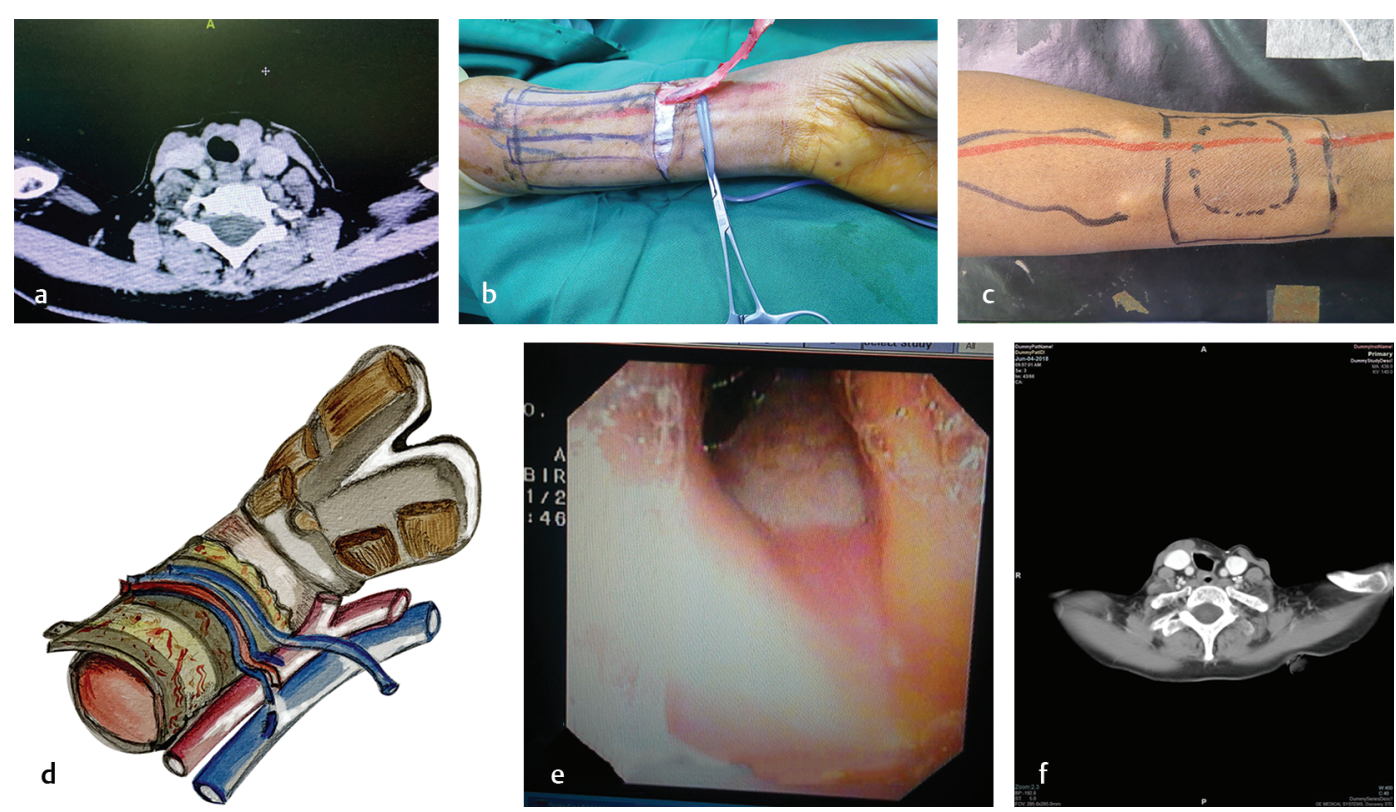

Fig. 1 (a) MRI of neck showing the lesion involving the trachea. (b) Fabrication of radial forearm flap with split rib grafts placed parallel to vascular pedicle. (c) Prefabricated flap prior to transfer. (d) Diagram illustrating the inset of the prefabricate flap. (e) Late postoperative endoscopic view of reconstructed prefabricated trachea. (f) CT scan of neck at 36 weeks postoperative, showing the well-maintained tracheal lumen with the overlying rib construct. 

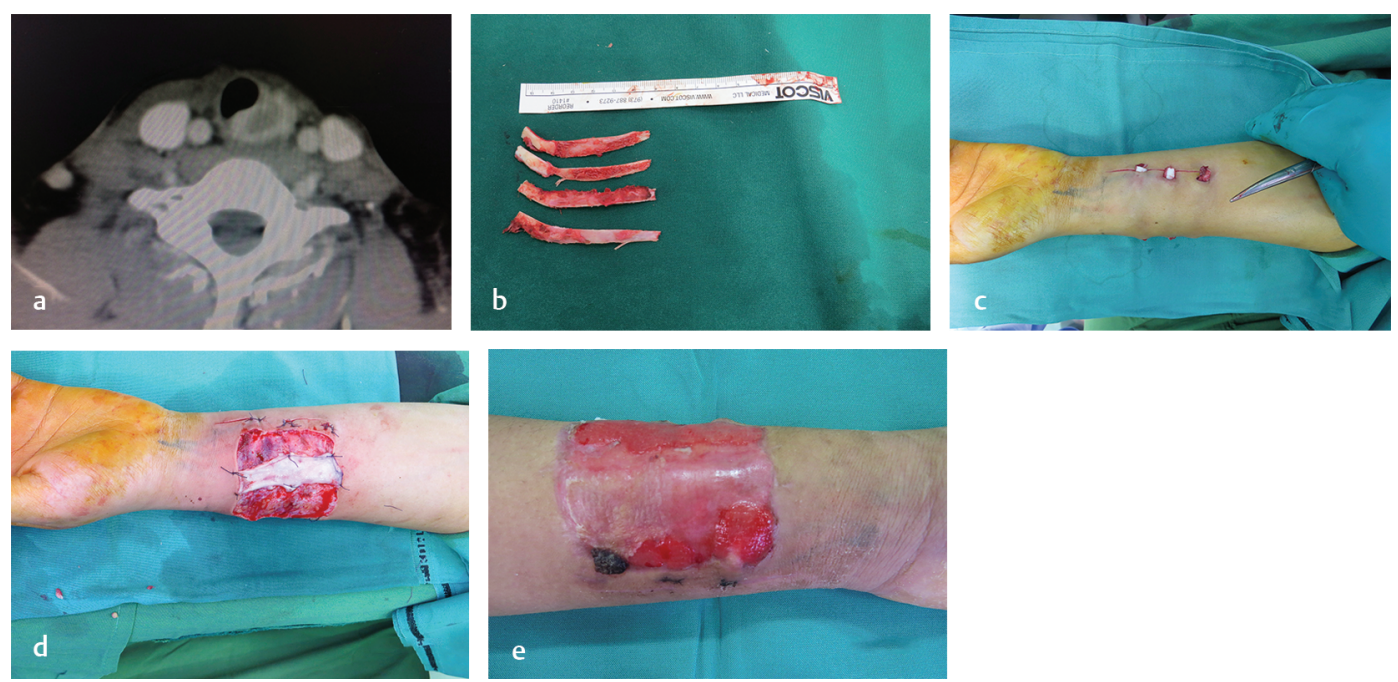

Fig. 2 (a) CT scan of neck showing a lesion involving the trachea as well as in proximity to the esophagus. (b) Harvested split rib grafts. (c) Design of the forearm construct with the split rib grafts placed transversely. (d) Prelamination of the construct using buccal mucosal grafts. (e) Prelaminated and prefabricated radial forearm flap prior to transfer.

Four weeks post the initial surgery, she was planned for the second stage after noting $80 \%$ take of the buccal mucosal graft ( - Fig. 2e). On exploration, a portion of 2 nd and 3 rd tracheal ring cartilage on left side seemed involved along with the constrictor of esophagus, which were dissected out keeping the tracheal mucosa intact. The cartilage defect was closed and covered with strap muscles, which were steps decided by the head and neck team.

The forearm construct was now redundant and needed excision. Replacing the defect with a skin graft would have left a scarred forearm; hence, a free superficial circumflex iliac artery perforator (SCIP) flap was used to resurface the forearm.

\section{Discussion}

Papillary carcinoma of the thyroid most commonly invades the strap muscles, with invasion of trachea accounting for $37 \%$. Survival is improved by complete surgical excision of the tumor. ${ }^{2}$ Nishida et al $^{3}$ examined 54 patients with stage II or higher tracheal invasion who underwent airway resection or subtotal resection without airway resection and noted a much higher recurrence rate (79\% vs. $8 \%$ ) and a shorter mean overall survival ( 1.5 years vs. 8.7 years) in the subtotal resection group.

For metastatic disease, surgery is combined with radioiodine therapy ${ }^{4}$

For locally aggressive papillary carcinoma, best outcomes are achieved with a complete surgical excision. Since the extent of the tumor was from the cricoid cartilage to the third tracheal ring, a complete excision would warrant excision of the cricoid cartilage. The surgical options with cricoid involvement were a total laryngectomy, which would result in permanent loss of her voice. Tracheal resection and reanastomosis were avoided because of involvement of only the anterior two third circumference of the trachea anteriorly, with functional vocal cords on laryngoscopy and without involvement of recurrent laryngeal nerve.

Tracheal reconstruction requires a vascularized noncollapsible semirigid construct, with a lining which has the ability to clear secretions.

Full-thickness tracheal defects up to $2 \mathrm{~cm}$ have been reported to be closed primarily. ${ }^{5}$ Reconstruction with a soft-tissue flap alone would inevitably collapse, with its consequences. Use of synthetic prosthesis and scaffolds lead to formation of obstructive granulation tissue and vascular erosion..$^{5}$ Use of porous scaffolds leads to overgrowth of scar tissue, resulting in obstruction and stenosis. ${ }^{5}$ Commonly acceptable conduits require a vascularized tissue with either an autologous or alloplastic support.

Various reconstructive options for the trachea have been reported, with most being single case reports. Use of vascularized flaps combined with external intraluminal stents has been reported. Intraluminal stenting with a prosthesis causes constant irritation and inflammatory responses, resulting in problematic secretions which, in turn, cause episodes of pneumonia and strictures. ${ }^{6}$ Beldholm et $\mathrm{al}^{6}$ in their case report used a combination of radial forearm flap with "ultraflex" self-expanding internal stent. The stent was composed of nickel and titanium alloy. However, the patient developed proximal stricture, sputum retention, and recurrent pneumonia.

A combination of alloplastic material and temporary internal stent was described by Adam et al $^{7}$ Four separate rings of biodegradative resorbable mesh made of glucose polymer were used to support the tube of radial skin island from the outside, which was followed by internal stenting for 6 months.

Yu et $\mathrm{al}^{8}$ successfully used a conduit composed of radial forearm flap for lining, with a combined $0.5 \mathrm{~mm}$ thick polymax resorbable mesh and $26 \mathrm{~mm}$ diameter premeshed vascular graft for rigid support. Titanium mesh has also been 
reported in experimental and clinical studies; however, it carries the risk of infection and extrusion. ${ }^{9}$

Walles and colleagues ${ }^{1}$ presented a very promising study of experimental generation of an engineered functional tissue and vascularized trachea. He however concluded that this study was far from clinical application and needed further experimental work. The use of autologous tissues has the advantage of resisting infection, extrusion, and rejection. Teng et $\mathrm{al}^{10}$ were the first to use prefabricated radial forearm flap with costal cartilage for anterior two thirds tracheal defects, as they placed the costal cartilage grafts in a transverse orientation. We used split rib grafts which have an inherent elasticity, allowing bending. The grafts were placed parallel to the vascular axis, presumably avoiding any risk to the perforators, and interposed between the fascia and skin. The whole construct was then placed horizontally. In the second patient, the split rib cartilage grafts were placed

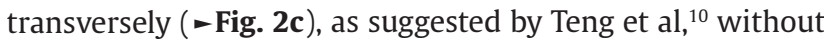
any compromise to the vascularity of the flap. They recommended that placing the support grafts in any direction interposed between the fascia and skin, with intervening areas of healthy tissue is possible.

Other innovative techniques have been reported. Olias et $\mathrm{al}^{11}$ described a successful three-stage reconstructive procedure for tracheal reconstruction. In the first stage, costal cartilage strips were sutured around a silicone tube and inserted into the radial forearm territory. In the second stage, after 10 weeks, the silicone tube was removed, and mucosal graft was wrapped around it. In the third stage, the microvascular transfer was performed.

To restore the tracheal continuity to its most physiologic condition, the soft-tissue lining needs to be supported by rigid materials that are placed around it, not inside the lumen. ${ }^{6}$ Ideally, the supporting material should already have been well-integrated with the soft-tissue lining at the time of reconstruction to avoid separation of the lining and airway collapse. This necessitates a two-stage reconstruction, and prefabrication of the forearm flap with the rib graft is ideal for this. Prefabrication allows combining different tissue types not present anatomically in the same region to make customized composite flaps with an independent vascularity.

In malignant lesions, there is an obvious disadvantage using staged procedures, with the risk of disease progression during the interval period. It may be possible to transfer a fabricated construct from the forearm in a single stage; however, if prelamination is planned, it would need to be staged.

Costal cartilage is rigid enough to prevent inward collapse, and it does not absorb over time. ${ }^{10}$ Patient on follow-up CT scan did not show resorption or collapse after 36 months (-Fig. 1). Radial forearm is preferred due to a stable blood supply and thin skin, making it pliable for prefabrication and also being amenable to prelamination.

There is a concern with regard to using skin for tracheal lining, with the possibility of shedding keratin debris in the bronchial system and the risk of airway irritation and infection. Yu et al in their article suggested that long-term follow-up of their patients tolerated skin lining well, with no such respiratory symptoms. ${ }^{12}$ Prelaminating the flap adds another dimension by adding a moist surface to the "inner" epithelium, avoiding the risk of desquamation and also the presence of hairs which may result in mucous trapping.

Free mucosal grafts harvested from the buccal mucosa was used in our second patient, where the construct was a composite of split rip grafts lined by prelaminated buccal mucosa, designed on the RAFF territory.

The extent and dimensions of the anticipated defect is dependent on the imaging. As observed in our second patient, close proximity with the trachea was misinterpreted. So, although the prefabricated and prelaminated construct was in the forearm, the actual excision resulted in a tracheal defect much smaller, which could be closed primarily, leaving the forearm construct redundant.

It is necessary to anticipate progression of disease between the two stages of reconstruction; hence, flaps of a larger dimension than the anticipated defect needs to be planned. It may be advisable to repeat an imaging prior to the second stage.

The ideal tracheal reconstruct needs to be reliable to be safely placed intrathoracically, as complications at this level would be life-threatening. Although a spectrum of surgical procedures has been explored, the prefabricated flaps are at present probably the most reliable option.

\section{Conclusion}

Staged radial forearm flap, prefabricated with costal cartilage, allowing the costal cartilage graft to get well-integrated with the soft-tissue of the flap prior to transfer is a feasible alternative for reconstruction of partial circumference tracheal defects. This may be further improved by prelaminating this composite with a mucosal graft, so as to mimic the tracheal defect to be replaced.

\section{Conflicts of Interest}

None declared.

\section{Acknowledgment}

No financial support received.

\section{References}

1 Walles T, Giere B, Hofmann M, et al. Experimental generation of a tissue-engineered functional and vascularized trachea. J Thorac Cardiovasc Surg 2004;128(6):900-906

2 McCaffrey TV, Bergstralh EJ, Hay ID. Locally invasive papillary thyroid carcinoma: 1940-1990. Head Neck 1994;16(2):165-172

3 Nishida T, Nakao K, Hamaji M. Differentiated thyroid carcinoma with airway invasion: indication for tracheal resection based on the extent of cancer invasion. J Thorac Cardiovasc Surg 1997;114(1):84-92 
4 Silberstein EB, Alavi A, Balon HR, et al. The SNMMI practice guideline for therapy of thyroid disease with 131I 3.0. J Nucl Med 2012;53(10):1633-1651

5 Belsey R. Resection and reconstruction of the intrathoracic trachea. Br J Surg 1950;38(150):200-205

6 Beldholm BR, Wilson MK, Gallagher RM, Caminer D, King MJ, Glanville A. Reconstruction of the trachea with a tubed radial forearm free flap. J Thorac Cardiovasc Surg 2003;126(2):545-550

7 Maciejewski A, Szymczyk C, Półtorak S, Grajek M. Tracheal reconstruction with the use of radial forearm free flap combined with biodegradative mesh suspension. Ann Thorac Surg 2009;87(2):608-610

8 Yu P, Clayman GL, Walsh GL. Human tracheal reconstruction with a composite radial forearm free flap and prosthesis. Ann Thorac Surg 2006;81(2):714-716
9 Haykal S, Salna M, Waddell TK, Hofer SO. Advances in tracheal reconstruction. Plast Reconstr Surg Glob Open 2014;2(7):e178

10 Teng MS, Malkin BD, Urken ML. Prefabricated composite free flaps for tracheal reconstruction: a new technique. Ann Otol Rhinol Laryngol 2005;114(11):822-826

11 Olias J, Millán G, da Costa D. Circumferential tracheal reconstruction for the functional treatment of airway compromise. Laryngoscope 2005;115(1):159-161

12 Yu P, Clayman GL, Walsh GL. Long-term outcomes of microsurgical reconstruction for large tracheal defects. Cancer 2011;117(4):802-808 